\title{
Strates
}

STRATES Matériaux pour la recherche en sciences sociales

8 | 1995

La question de l'environnement: recherches parallèles en Espagne et en France

\section{Les métiers de l'environnement}

Nathalie Le Coz

\section{OpenEdition}

Journals

Édition électronique

URL : http://journals.openedition.org/strates/1079

DOI : $10.4000 /$ strates. 1079

ISSN : $1777-5442$

Éditeur

Laboratoire Ladyss

Édition imprimée

Date de publication : 30 juin 1995

ISSN : 0768-8067

Référence électronique

Nathalie Le Coz, «Les métiers de l'environnement », Strates [En ligne], 8 | 1995, mis en ligne le 20

décembre 2005, consulté le 08 septembre 2020. URL : http://journals.openedition.org/strates/1079 ;

DOI : https://doi.org/10.4000/strates. 1079

Ce document a été généré automatiquement le 8 septembre 2020

Tous droits réservés 


\title{
Les métiers de l'environnement
}

\author{
Nathalie Le Coz
}

Des métiers qui n'existent pas officiellement

1 On ne peut que constater l'absence de la rubrique métiers de l'environnement dans les nomenclatures officielles des métiers, que ce soit dans celle de l'Agence Nationale pour l'Emploi (A.N.P.E.), ou dans celles du Centre d'Etudes et de Recherches sur les Emplois et les Qualifications (C.E.R.E.Q.), des ministères de l'Education Nationale, du Travail, de l'Industrie... Restait donc jusque-là l'inventaire du ministère de l'Environnement, dont les catégories renvoient aux domaines dont l'institution a la charge.

La cause en incombe à l'inexistence d'appellations officielles correspondant à des diplômes donnés, ou recouvrant une activité précise. Cela tient en partie à l'apparition récente des activités pour l'environnement dans les sphères du social et du culturel, mais aussi et surtout à des caractéristiques structurelles : l'environnement étant perçu comme interdisciplinaire, il tend à investir des activités économiques traditionnellement reconnues. Des qualifications et des métiers précis qui se situent dans le champ de l'environnement, et ceci quelque soit l'activité, tentent donc actuellement d'être déterminés.

3 Pour pallier cette absence officielle, l'Institut Français de l'Environnement (I.F.E.N.) a mis en place en 1993 un groupe de travail en liaison avec l'I.N.S.E.E., afin de définir les critères permettant d'établir une nomenclature des métiers, des emplois et des activités « environnement ", à partir des nomenclatures déjà existantes.

4 Pourtant nous pouvons nous interroger sur la pertinence de la construction et de l'utilisation d'une nomenclature, dès lors que l'identité professionnelle d'un individu s'appréhende de moins en moins aujourd'hui sur la base d'un emploi, d'une profession, ou d'un statut unique dans une perspective plus ou moins figée. Longtemps perçu comme un enjeu déterminant dans la structuration des identités sociales, l'emploi permettait à l'individu d'être socialisé et reconnu, d'être partie prenante d'un groupe. Mais aujourd'hui où s'imposent la multiplicité des situations professionnelles et une évolution des tâches dans le domaine qui nous concerne, on peut se demander si le paradigme de l'emploi n'est pas ébranlé, si les catégories juridiques, statistiques et les représentations sociales ne sont pas altérées. Cette interrogation est d'autant plus forte 
que les concepts d'emploi « environnement » et de métier de l'environnement sont des concepts dynamiques qui fluctuent au cours du temps, aussi bien dans leur contenu quantitatif que qualitatif et social. Mais si ces deux notions d'emploi et de métier sont confrontées à des problèmes de définitions liés à la perception même du champ de l'environnement, la notion de métier renvoie plus spécifiquement encore à un problème de terminologie. Peut-on véritablement parler de «métiers de l'environnement »?

Un contexte dynamique

5 Comprendre l'interrogation, somme toute récente, sur ces métiers et leur identification, ne peut se faire sans resituer celle-ci dans un contexte plus global dans lequel interviennent conjointement la réflexion sur les emplois et l'émergence de politiques spécifiques dans ce domaine. Deux facteurs déterminants et interactifs sont à l'origine de la multiplication des inventaires des métiers. de l'environnement.

6 Tout d'abord, ces derniers s'inscrivent dans une réflexion globale portant sur les emplois «environnement» qui trouve sa source dans un débat atteignant son paroxysme dans les années 1970: l'environnement constitue-t-il une menace pour l'emploi en raison de fermetures d'usines ou de la contraction de l'activité économique, ou bien, au contraire, une source d'emplois ? Durant cette période, les programmes de protection de l'environnement sont fortement contestés par certains milieux industriels et syndicaux, notamment lors des chocs pétroliers de 1974 et 1979. Un débat qui intervient aussi face à la montée des sensibilités écologiques: ces dernières opposent alors croissance économique et préservation de la nature, en réponse à la prise de conscience de la multiplication des pollutions et du gaspillage des ressources naturelles. De même, c'est à la fin de cette période charnière que les politiques de l'environnement ne se limitent plus à la lutte contre les pollutions de l'air et de l'eau. Elles intègrent en 1976 la protection de la nature, et commencent à investir le domaine de l'aménagement.

7 Au niveau de l'OCDE, des études économétriques sur l'incidence des politiques de l'environnement sur l'emploi se développent alors (conférences de 1978 et 1984), tandis qu'en France des études sur l'évaluation du nombre d'emplois directement liés à l'environnement $(1976,1979)$, et indirectement liés (1981) apparaissent ${ }^{1}$.

8 Parallèlement, des politiques spécifiques d'emplois liés à l'environnement se mettent progressivement en place, suite à un débat reformulé, par rapport à celui des années 1970, en ces termes : «La question est non seulement de faire en sorte que les politiques de l'environnement n'entraînent pas d'effets négatifs sur l'emploi mais qu'elles contribuent le plus efficacement possible à la création d'emplois $»^{2}$. Véritablement instaurées à partir du début des années 1980, ces politiques ont trois objectifs: développer de nouvelles productions, freiner le développement du chômage, et promouvoir l'intégration sociale et/ou professionnelle des jeunes.

9 C'est dans ce contexte que les inventaires des métiers vont progressivement surgirent. Le premier, celui de Anne Galey, paraît dans une période où émergent le programme "emploi-environnement ${ }^{3}$ (1982) et surtout la politique des TUC-Environnement ${ }^{4}$ (1984). Il faudra ensuite attendre les années 1992-94, durant lesquelles la politique des CES-Environnement ${ }^{5}$ se substitue à la précédente, pour voir paraître des inventaires de plus en plus nombreux. Leur multiplication devient le reflet d'une réflexion qui se focalise sur un écueil méthodologique contre lequel butent toutes les tentatives 
d'évaluation et de prospective du nombre d'emplois: qu'entend-on par emploi « environnement»?

10 Se développent alors des études sur la nature des emplois dans les différents domaines sectoriels, tels que l'eau et les déchets, deux domaines qui sont l'objet de nombreuses directives européennes et de lois nationales. De même, des structures de réflexion se mettent en place, de nouvelles formations s'instaurent, des colloques en débattent, à l'exemple du forum d'Avignon sur le thème des «emplois cadre environnement dans l'Europe du Grand Marché » (juin 1990).

11 C'est donc bien à partir des années 1990 que l'on s'interroge réellement sur ce que sont les emplois, les métiers, et les activités de l'environnement. Une interrogation qui ne porte plus seulement sur une connaissance de leur portée économique, mais aussi de ce qu'ils sont exactement. Les auteurs des guides et inventaires des métiers de l'environnement se veulent chacun participer au débat grandissant, bien que ces derniers soient difficiles à établir tant les frontières qui bordent ce secteur sont mal définies.

Vers une légitimité des métiers de l'environnement?

12 Réalisés par le ministère de l'Environnement et surtout par des journalistes intervenant ou non dans des revues spécialisées, ces guides s'adressent aux futurs "professionnels", et voudraient répondre aux questions des "jeunes" en quête d'informations, dont les candidatures dans ce domaine se multiplient.

13 Huit inventaires ou guides sont parus à ce jour (cf. liste in fine). Leur nombre important, et surtout les désaccords qui subsistent de l'un à l'autre quant à la prise en compte de certaines activités, de certaines "professions ", nous ont amenée à établir un tableau de croisement de ces guides ou inventaires, ou du moins de ceux présentant une liste détaillée des «métiers » de l'environnement. Ce tableau permet de mettre en évidence un degré de légitimité des métiers selon qu'ils apparaissent dans tous les inventaires retenus ou non.

14 Ainsi certains métiers sont les plus légitimés puisqu'ils sont les plus récursifs d'un inventaire à l'autre, d'une colonne à l'autre. Repris par les différents auteurs, ils paraissent aller de soi (ils sont figurés en gras dans le tableau comparatif). Néanmoins, des variantes terminologiques et de qualification contribuent à atténuer cette récurrence. L'exemple du domaine acoustique est révélateur. Si certains ne citent que les acousticiens, ou bien les acousticiens et les techniciens en acoustique, d'autres ne citeront que les techniciens et les ingénieurs dans ce même domaine. Difficile de savoir alors sous quel « terme exact » ce « métier » peut aller de soi.

15 L'organisation du tableau en six colonnes selon un ordre chronologique de parution des inventaires permet aussi de dégager une évolution du degré de légitimité dans le temps. Certaines "familles de métiers" se trouvent par exemple grossies par l'intégration de nouveaux «termes »: les secteurs des déchets et de l'assainissementépuration sont significatifs. Ces derniers apparaissent surtout à partir de l'inventaire du ministère de l'Environnement, en 1992. De même, l'égoutier et le rudologue, par exemple, ne sont cités que depuis 1993 . La diversification des métiers qui transparait dans les inventaires postérieurs à 1990 ne peut qu'être consécutive à une meilleure connaissance de ces derniers en raison du récent développement des études sectorielles. Enfin, on peut noter l'intégration récente des «métiers" concernant les barrages et les canaux, relevés dans l'inventaire de Françoise Chirot et Anne Galey. Bien au contraire, les «métiers de l'équitation » perdent de leur intérêt dans le domaine qui 
nous intéresse, à l'égal, entre autres, des apiculteurs, des aviculteurs, des héliculteurs... Ils n'apparaissent plus comme des métiers de l'environnement à partir de l'inventaire de 1992.

Consensus et désaccords surgissant d'un inventaire à l'autre trouvent tout d'abord leur origine dans la nécessité et la difficulté de prendre en compte des domaines non directement liés à l'environnement mais qui l'intègrent de plus en plus, englobant et dépassant les domaines sectoriels (par thèmes environnementaux) correspondant à une définition technicienne ou réglementaire de l'environnement. Des quiproquos apparaissent alors entre la tâche première de certaines activités et "l'utilité sociétale » de l'environnement. Enfin, la question de la légitimité ou de l'illégitimité de la dénomination "métier de l'environnement" se pose à travers la réalité du terme même de métier.

Des métiers au conditionnel

17 C'est l'évolution du concept même d'environnement, de son contenu, retenu par les politiques, les réglementations, les sensibilités et les préoccupations environnementales de la société, qui rend très ambigus certains métiers. Parce qu'il apporte à des professions qui ne lui sont pas spécifiques une dimension supplémentaire, l'environnement impose une définition qui doit être appréhendée le plus souvent en termes d'évolution des tâches. Ces dernières s'inscriraient alors dans le champ de l'environnement selon les objectifs dans lesquels elles s'intègrent (protection, sauvegarde, gestion, entretien...), et selon «l'utilité sociétale» de l'environnement (production, loisirs, esthétisme...).

18 Ainsi, si l'on parle des activités qui concernent «directement la protection de l'environnement ", n'est-ce pas un jugement hâtif de considérer le secteur de l'eau, par exemple, comme le principal pourvoyeur d'emplois, puisqu'une part importante est occupée par la mobilisation, l'exploitation, de la ressource ? En ce sens, les "métiers » de ce secteur apparaissent peu cités dans le domaine qui nous concerne.

Il en va tout autrement en ce qui concerne les vétérinaires, que l'on retrouve dans la plupart des inventaires. Néanmoins, pour Véronique Boisdon ${ }^{6}$, leur titre de spécialistes de l'environnement se trouve justifié seulement pour «ceux qui exercent leur art dans les parcs naturels ou qui participent à la préservation d'une espèce menacée ». En fait, certains métiers dépendent surtout des conditions d'exercice. Horticulteurs, pépiniéristes, paysagistes et bûcherons apparaissent ainsi parmi les métiers les plus légitimes. Véronique Boisdon soulève le cas des horticulteurs dont le rôle est perçu dans le champ de l'environnement en se modifiant, "lorsqu'ils sont chargés de mettre en valeur le patrimoine floral d'un parc ou d'un jardin ». Il en va de même pour les paysagistes qui « ont maintenant largement dépassé le stade de la floraison des jardins publics ". C'est bien à travers une évolution des pratiques, générée par un changement des mentalités consécutif à de nouvelles contraintes réglementaires et à une demande sociale qui s'amplifie quantitativement et qualitativement, que les « métiers forestiers » sont très largement cités. Bien que le bûcheron ou le responsable d'exploitation considère la forêt d'abord comme une ressource économique, et bien que les agents de l'office National des Forêts aient pour tâche première la production de bois, sans rapport donc avec la protection de l'environnement, ces métiers tendent cependant à être perçus de plus en plus dans le champ de l'environnement par la dimension "aménagement et entretien de l'espace » qui leur est progressivement conférée. Il n'est plus seulement question de protéger l'environnement, mais aussi de gérer et d'entretenir. Ce «reprofilement» 
touche tout autant la profession agricole, quoique les agriculteurs «biologiques" demeurent les plus légitimes à travers les inventaires.

La complexité de la définition des métiers de l'environnement va donc bien au-delà du débat institué par la confrontation entre deux opinions. A savoir que pour les uns, l'environnement est une formation spécifique. Il s'agit alors de métiers « $100 \%$ environnement ", résultant d'une qualification professionnelle caractéristique. Sont le plus souvent cités ici les éco-conseillers, les éco-juristes, les spécialistes d'études d'impact ou audit environnemental, le responsable déchets... Pour d'autres, l'environnement n'est qu'une question de formations complémentaires. Il s'agit alors de métiers « repeints en vert ", tel que l'éco-communicant. Dans ce cas, la composante " environnementale » de l'emploi devient difficile à identifier. Ainsi le chimiste chargé du contrôle des pollutions, cité dans la plupart des inventaires, est-il d'abord un chimiste ou un " environnementalist » ?

21 Ce débat ne doit plus seulement être appréhendé autour de la seule notion d'environnement et de la prise en compte des domaines sectoriels et transversaux. Il laisse entrevoir aussi, et peut-être surtout, la difficulté d'employer le terme de métier. En fait, on ne sait plus trop bien s'il faut parler de profils, de missions, de fonctions, d'emplois, de professions, de qualifications... dès lors que des pratiques nouvelles, et corollairement de nouvelles compétences, une nouvelle philosophie du travail, surgissent.

Des bons termes au bon langage

Il faut savoir que c'est à travers une définition des métiers à forte connotation de manuel, d'artistique et d'artisanat que Anne Galey intégrait dans son inventaire de 1984 le charpentier, le tailleur de pierre, le sculpteur sur bois, le ferronnier d'art, le luthier, le menuisier, le fondeur de cloches... Mais aujourd'hui, dans un contexte et dans un domaine où l'on ressent la nécessité que "l'image du petit et du sale boulot » évolue pour aboutir à une "image de professionnalisme et de qualification", on peut se demander si la définition ne repose pas davantage sur celle, implicite, du "professionnel de l'environnement ", tendant à légitimer socialement un travail dans un secteur non encore reconnu comme un secteur d'activité à part entière. En effet, si l'on se réfère à la définition de "profession ", celle-ci renvoie à un métier ayant un certain prestige par son caractère intellectuel ou artistique, par la position sociale de ceux qui l'exercent. Ainsi, des responsables espaces verts, et des «métiers » du secteur des déchets, par exemple, ne cherchent-ils pas aujourd'hui à revendiquer une professionnalisation de leur travail? Les mots emploi, profession et métier, le plus souvent employés comme équivalents, recouvrent en fait des réalités différentes consécutives à un désaccord qualitatif.

D'après l'Institut Français de l'Environnement ${ }^{7}$, cette définition, reposant sur celle du professionnel de l'environnement, correspond le plus souvent à une distribution des qualifications sur laquelle, en réalité, reposent les deux types de domaines d'activité caractérisant la définition des emplois environnement (domaines sectoriels et transversaux). Ces «deux univers professionnels» opposent alors entre eux des définitions qui renvoient à des hiérarchies de valeurs et à des sources de légitimité différentes. On retombe ici sur le débat précédemment explicité.

Faut-il alors parler de nouveaux emplois, de nouveaux métiers... ou bien simplement de nouvelles tâches, d'un travail émergent? Pour certains, la prise en compte de l'environnement se traduira par un enrichissement des compétences dans des postes 
existants, modifiant la dénomination de ces derniers: il s'agit bien alors d'une évolution des tâches, transformant des fonctions existantes, surtout dans les entreprises où les définitions de fonctions évoluent, donnant naissance à une terminologie spécifique. L'éco-conseiller, l'éco-communicant, l'éco-toxicologue ou encore le responsable environnement en entreprise sont donnés comme exemples. L'environnement est alors perçu comme un facteur de progression professionnelle et d'enrichissement des tâches. En conséquence, certains parleront de profils ou de qualifications, alors qu'ils voudraient parler de missions/fonctions, s'agissant le plus souvent d'emplois nouveaux que de métiers.

Mais si l'environnement est bien un domaine où émergent de nouvelles tâches, pour d'autres il s'agirait aussi "d'inventer des modes d'exercice contemporain de métiers traditionnels... tenant compte des attentes de la société contemporaine »" En tout état de cause, certains inventaires, aujourd'hui, font état de l'apparition de " nouveaux métiers potentiels ", ou plutôt de nouvelles fonctions, qui se révèlent à juste titre n'être encore que de nouvelles formations face à l'absence d'appellations officielles. Ainsi figurent les éco-conseillers et les éco-interprètes. Les premiers sont nés d'une expérience pilote de formation testée à Hambourg en 1985, puis étendue à la France en 1987, avec la création de l'Institut pour le Conseil en Environnement à Strasbourg. Les seconds, qui sont surtout des animateurs, sont nés d'une formation principalement dispensée depuis 1989 par le Centre Permanent d'Initiation à l'Environnement de Franche-Comté. Dans le domaine de l'animation, nombreuses sont les formations spécifiques progressivement mises en place, avec des diplômes à option "environnement » homologués par le ministère de la Jeunesse et des Sports.

Un enjeu réel s'impose : constituer des référentiels métiers, et lever le flou entourant la définition des métiers de l'environnement, ou du moins des emplois. Ceci est une condition sine qua non face à l'émergence de nombreuses formations et revendications de professionnalisation dans ce domaine. C'est une nécessité à laquelle doit tenir compte la récente politique des «emplois verts » mise en place par les ministères de l'Environnement et du Travail, de l'Emploi et de la Formation Professionnelle. La pérennité des emplois issus de Contrats Emplois Solidarité dans le domaine de l'environnement ne peut être effective que si des référentiels métiers sont constitués.

Dans ce débat grandissant, une recherche partant d'une approche par l'espace géographique s'avère très pertinente, apportant une dimension supplémentaire en confrontant la problématique des emplois et des métiers à une réalité, à une dynamique locale. Une telle recherche donne toute son importance à la question du rôle de l'espace dans la constitution et le vécu de ces "nouveaux emplois", de ce travail émergent, et à la question de savoir qui sur le terrain s'y engage, de quelle manière, en fonction de quels besoins spécifiques. De plus, face à l'absence d'un consensus autour d'une seule définition, et parce que le concept même d'environnement est difficilement "appréhendable » d'un commun accord de par sa polysémie et sa subjectivité, la nécessité est ressentie de tenir compte des définitions d'usage. Les perceptions sociales ne peuvent être que résolument prises en compte, à savoir ce que les acteurs qui gèrent des emplois et qui pratiquent un travail dans ce domaine considèrent eux-mêmes comme relevant du champ de l'environnement.

Cette approche par l'espace et le local devient déterminante en tant que support de la réflexion dès lors qu'elle permet de s'interroger véritablement sur la nature du travail, des emplois et des tâches. Une telle réflexion tient compte de l'impact des politiques 
spécifiques (politiques des "emplois verts", et applications des directives agrienvironnementales notamment) sur le développement local et de la mobilisation des acteurs. L'identification de l'ensemble des intervenants et du rôle de chacun devient alors un réel enjeu, leurs perceptions et leurs stratégies étant directement confrontées à leur « vécu " par rapport à un espace géographique donné, par rapport à la diversité des situations locales induite par celle du milieu physique et des contextes socioéconomique, démographique, politique, culturel, ainsi que des idéologies. Une diversité et des contextes qui ne peuvent que dénoter des besoins ressentis, tant au niveau de l'adaptation des formations et des emplois, qu'à celui des différentes structures présentes, à travers la mise en place de systèmes organisationnels .

Le rôle de l'espace géographique pourra être mis en exergue par le choix des HautesVosges en tant que terrain d'étude, tenant compte de la présence du Parc Naturel Régional des Ballons des Vosges, et de la contiguïté entre trois régions (Alsace, Franche-Comté, Lorraine), et quatre départements9.

\section{BIBLIOGRAPHIE}

\section{Guides et inventaires des métiers de l'environnement par ordre de parution}

GALEY A.. \& CALENDRIER E. (1984), Les Métiers de la nature et de l'environnement, Paris, Stock, coll. Laurence Pernoud.

RAFFOUL M. (1990), Guide des métiers : l'environnement, Paris, Bayard, coll. CIDJ Phosphore.

Inventaire national des formations et métiers de l'environnement (1992), Paris, ministère de l'Environnement, Délégation à la Qualité de la Vie.

MABIT M.(1993), Les Métiers de l'environnement : gagner sa vie en protégeant la vie, Nantes, Ed. Opéra.

PENA J-M. (1993), Les Métiers de l'environnement, Coll. l'Etudiant Pratique, série : vocation.

BOISDON V. (1994) Les Métiers de l'environnement, Guides du Monde de l'Education, Le MondeEditions, Marabout.

LE SEIGNEUR V.J. (1994), Le Guide des métiers et des formations de l'environnement, Paris, Dunod.

CHIROT F.\& GALEY A. (1994). Guide des métiers verts, avec ou sans diplôme, (en collaboration avec la Fondation Ushuaïa et l'association Emploi Nature) ; Paris, Editions Sang de la Terre.

\section{NOTES}

1. Cf. Tableau chronologique in Le Coz N. (1994), Emplois et situations de travail dans le domaine de l'environnement, Mémoire de DEA, Université de Paris I.

2. «Environnement et emploi », in Aménagement et nature, 114, Les emplois de l'environnement, 1994, pp. 59-64 (article extrait des Données économiques de l'environnement, 1993). 
3. Ce programme devait stimuler des « micro-projets créateurs d'emplois au niveau local », en favorisant « l'innovation sociale », donnant une place particulière aux associations et aux entreprises intermédiaires. Cette opération devait surtout permettre la mise en place de moyens spécifiques de formation professionnelle pour l'élevage, la conduite de petites stations d'épuration, ou l'animation « nature » dans les villes.

4. La politique des TUC (Travaux d'Utilité Collective) Environnement devait aussi favoriser l'insertion professionnelle « dans les métiers de l'environnement » (élagueurs, pépinièristes, gardes-rivières...). Dans cet objectif a été lancée en 1988 l'action « 1000 kilomètres de rivières » qui a connu de grandes disparités régionales consécutives à une mobilisation différente entre le thème de la gestion des rivières et la problématique TUC. Mais surtout ces « chantiers » ont été très critiqués, jugés trop aléatoires, ponctuels et dispersés.

5. Le dispositif des CES (Contrats Emploi Solidarité) Environnement se met en place en 1992-93 à travers la coordination d'organismes d'insertion par l'environnement. Cette action vient d'être recentrée par le biais d'une incitation financière à la création d'emplois : dispositif des « emplois verts ». Des aides sont apportées pour le démarrage de projets permettant la création de cinq emplois (bénéficiant aux collectivités territoriales et aux associations), et pour le recrutement d'un chef d'équipe encadrant cinq emplois nouvellement créés (bénéficiant aux seules associations). Dans le cadre de ce dispositif, une mobilisation a été demandée aux établissements publics sous tutelle du ministère de l'Environnement.

6. Auteur d'un guide non pris en compte dans le tableau comparatif.

7. Structure et profils des emplois environnement en France, IFEN, Travaux et recherches, 1 , avril 1993.

8. Turquin O. (1994), « Les emplois verts en zone rurale : mirage ou miracle? » in Aménagement et nature, op. cit. , pp. 13-23.

9. Le Coz N., op. cit.

\section{RÉSUMÉS}

La recherche, somme toute récente, de ce que sont les métiers de l'environnement et de leur reconnaissance sociale et officielle se trouve confrontée à des problèmes de définitions consécutifs à la fois aux divergences de perception du champ de l'environnement et à une remise en question de la notion même de métier.

\section{What about the jobs dealing with environment?}

The jobs, trades and occupations dealing with environment matters have only been identified very recently. Their social acceptance and official recognition are depending on a great variety of viewpoints concerning how environmental question are perceived and, besides, how the mere motion of work is nowadays debated.

Los oficios relacionados con el medio ambiente. 
La investigación, en resumidas cuentas muy reciente, en lo que se refiere a los oficios relacionados con el medio ambiente y su reconocimiento social y oficial, se encuentra confrontada con problemas de definición, derivados de las divergencias en la percepción del sector del medio ambiente e inclusive de una nueva discusión sobre la noción misma de oficio.

INDEX

Mots-clés : Environnement, Métiers, Professions, Emploi, Inventaires

Keywords : environment, Jobs, Trades, Occupations, Employment, Inventories

Palabras claves : medio ambiente, Oficios, Profesiones, Empleo, Inventarios

\section{AUTEUR}

\section{NATHALIE LE COZ}

Doctorante en géographie à l'Université Paris I. Rattachée au laboratoire STRATES, elle travaille actuellement sur les emplois et les métiers de l'environnement dans les régions de l'Est de la France. 